\title{
Statin Therapy in the Management of Diabetes Mellitus; How Relevant?
}

\author{
Fasanmade Olufemi Adetola and Odeniyi Ifedayo Adeola \\ Department of Medicine, Faculty of Clinical Sciences, \\ College of Medicine, University of Lagos, PMB 12003, Surulere, Lagos, Nigeria
}

Received 2012-05-18, Revised 2013-01-11; Accepted 2013-03-25

\begin{abstract}
Diabetes is associated with the development of premature cardiovascular disease. There have been many clinical trials of statin treatment conducted in patients with Coronary Heart Disease (CHD). Taking a closer look at the subset of those patients with diabetes mellitus puts no doubt on the beneficial effect of lowering cholesterol with statin in these patients. Earlier studies done dwell more on cholesterol reduction with statins in patients with CHD but without diabetes hence no strong supporting evidence was there to justify the use of statins in diabetic patients without cardiovascular disease. We search medline for studies on cholesterol reduction and cardiovascular risk reduction in diabetic patients. We found three secondary prevention trials (4S, CARE and LIPID); four primary prevention trials (ASCOT-LLA, CARDS, WOSCOPS and TexCaps); two mixed prevention trials (HPS and ASPEN); three trials (PROVE-IT, TNT and A to Z) that seek to evaluate whether intensive or moderate lipid lowering with statins will prevent cardiovascular risk and to what extent. Treatment decisions should be based on the reduction of absolute risk and not on the reduction in relative risk. Patients suffering from diabetes mellitus will incur a huge cost if they were to be commenced on statin from the day the diagnosis of diabetes was made. With the rising incidence of diabetes worldwide, statins should be targeted to those patients with diabetes mellitus who are most likely to benefit from it. Type 2 DM patients with metabolic syndrome or those with mild to moderate cardiovascular risk will benefit from statin therapy. Clinical benefits stand to be derived from statin therapy if moderate reduction of risk can be achieved in patients that are classified as having high cardiovascular risk. What this means is that all patients eligible to be enrolled in the HPS or CARDS study should be on statin treatment.
\end{abstract}

Keywords: Cardiovascular Disease (CVD), Collaborative Atorvastatin Diabetes Study (CARDS), Coronary Heart Disease (CHD), Cholesterol and Recurrent Events Study (CARE)

\section{INTRODUCTION}

New figures indicate that the number of people living with diabetes is expected to rise from 366 million in 2011 to 552 million by 2030, if no urgent action is taken (IDF, 2012). This equates to approximately three new cases every ten seconds or almost ten million per year. IDF also estimates that as many as 183 million people are unaware that they have diabetes (IDF, 2012). The increasing prevalence of obesity and the metabolic syndrome contribute to the increase in frequency of diabetes
(Mokdad et al., 2003; Ford et al., 2002). There is no doubt whatsoever that the use of statins in people with diabetes mellitus is full of benefits. What have not been categorically stated is whether all diabetic patients should be treated with a statin. This review looked at evidences that exist in literature for the use of statins in patients with diabetes from randomised, controlled trials.

Cardiovascular Disease (CVD) is the leading causes of mortality and morbidity for persons with diabetes mellitus. Diabetes mellitus is an independent risk factor for the development of macrovascular complications.

Corresponding Author: Fasanmade Olufemi Adetola, Department of Medicine, Faculty of Clinical Sciences, College of Medicine, University of Lagos, PMB 12003, Surulere, Lagos, Nigeria Tel: +2348033008127 
The presence of hypertension and dyslipidemia as comorbidity in individuals with diabetes mellitus make the risk higher (Turner et al., 1998). The U.K. Prospective Diabetes Study (UKPDS) evaluated baseline risk factors for coronary artery disease in patients with newly diagnosed diabetes without evidence of vascular disease. The modifiable risk factors in this study are hyperglyceamia, hypertension and dsylipidaemia. The contribution of each of these risk factors to the development of coronary heart disease was assessed. The estimated hazard ratios for the upper third relative to the lower third were 2.26 (95\% confidence interval 1.70 to 3.00) for low density lipoprotein cholesterol, 0.55 (0.41 to 0.73 ) for high density lipoprotein cholesterol, 1.52 (1.15 to 2.01) for haemoglobin A1c and 1.82 (1.34 to 2.47) for systolic blood pressure (Turner et al., 1998). The major contributor to increased CHD risk in patients with diabetes mellitus is dyslipidemia, (specifically LDL cholesterol) (Turner et al., 1998; Haffner, 1998). The most common pattern of dyslipidemia in diabetic patients is elevated triglyceride levels and decreased HDL cholesterol levels (Haffner, 1998; Ogbera et al., 2009; Haffner, 2004). Some researchers have posited that the quality of LDL also play a role in the risk of cardiovascular events. The LDL particles in patients with diabetes mellitus has been found to be smaller and denser and thus make these patients prone to oxidation and increased risk of cardiovascular events (Haffner, 2004; Graaf et al., 1991). It is known that cardiovascular disease risk may even be present in the period of prediabetes or at the time of diagnosis, the so called ticking clock hypothesis of Haffner et al. (1990).

\subsection{Secondary Prevention Trials Involving Diabetic Patients}

The Scandinavian Simvastatin Survival Study (4S) (SSSS, 1994) was the first trial-based evidence that cholesterol lowering reduced the risk of major CHD events and other atherosclerotic events in diabetic patients. In the Scandinavian Simvastatin Survival Study (4S) a post-hoc subgroup analysis of the data from 202 diabetic patients there was a larger reduction in the primary end point of all-cause mortality in this group when compared to the whole trial population. However this did not reach statistical significance (RR $0.57, \mathrm{p}=$ 0.087). The small size of the subgroup may be responsible for this. There was a significant reduction in major coronary events from 44 events in the placebo group to 24 in the diabetic group ( $R R$ 0.45, p = 0.002) (SSSS, 1994; Pyorala et al., 1997).

In the Cholesterol and Recurrent Events Study (CARE) (Sacks et al., 1996), 586 patients were in the diabetic subgroup. When compared with those in the non-diabetic group, those in diabetic group had higher level of obesity, majority of them have hypertension and they were older. However their Low-Density Lipoprotein (LDL) cholesterol levels was similar (Goldberg et al., 1998). Treatment with pravastatin resulted in an insignificant reduction in the primary end point of death from coronary heart disease or non-fatal MI (RR $0.87, p$ $=\mathrm{ns})$. A significant reduction was observed in an extended primary end point, however, that included revascularisations $(\mathrm{RR} 0.75, \mathrm{p}=0.05)$, with a larger absolute risk reduction of coronary events in the diabetic subgroup than in the study population as a whole (8.1 Vs. 5.2\%), reflecting the fact that this subgroup had a higher level of risk in general (Goldberg et al., 1998).

In the diabetic subgroup of the Long-term Intervention with Pravastatin in Ischemic Disease (LIPID) Study (LIPID, 1998), 1,077 subjects with type 2 diabetes were included in the cohort (Keech et al., 2003). There was a $19 \%$ reduction in major coronary events in the diabetic subgroup however, this was not significant when compared to placebo (23.4 Vs. $19.6 \% \mathrm{p}=0.11)$. The absolute risk of a first CHD event was higher in the diabetes subgroup (23.4 Vs. 14.5\%). In a period of over six years, one major CHD-related event was prevented in 18 patients in the diabetes subgroup with prevastatin therapy (Keech et al., 2003).

\subsection{Primary Prevention Trials Involving Diabetic Patients}

The West of Scotland Coronary Prevention Study (WOSCOPS) involving only 76 known diabetic patients (Shepherd et al., 1995) and the Airforce/Texas Coronary Atherosclerosis Prevention Study (AFCAPS/TexCAPS) study had 155 people with diabetes (Downs et al., 1998) lent credence to the fact that lowering the cholestrol level in patient with elevated cholesterol has beneficial effect in primary prevention of cardiovascular event.

The Anglo-Scandinavian Cardiac Outcomes Trial Lipid-Lowering Arm (ASCOT-LLA) was a lipidlowering arm of a hypertension study, comparing $10 \mathrm{mg}$ of atorvastatin in patients with hypertension but no previous CHD (Sever et al., 2003). Patients in ASCOTLLA had uncontrolled hypertension and three or more pre-specified cardiovascular risk factors including type 2 diabetes. Their average total cholesterol was $5.5 \mathrm{mmol}$ 
$\mathrm{L}^{-1}$. The primary end point were non-fatal MI and fatal CHD. Treatment was stopped after a median follow-up of 3.3 years. This was because 100 primary events have been recorded in the atorvastatin group and 154 events have been recorded in the placebo group. This represents $36 \%$ reduction in primary end point (Hazard Ratio (HR) was 0.64 [95\% CI $0.50-0.83]$; $\mathrm{p}=0.0005)$. There was a $21 \%$ reduction in total cardiovascular events (389 vs 486, 0.79 [95\% CI 0.69-0.90], $\mathrm{p}=0.0005$ ) (Sever et al., 2003). In the diabetic subgroup, a $16 \%$ risk reduction was recorded. This was not statistically significant. The early termination of the trial puts a limitation on the power of any test to compare effect of statins with those in the placebo group since the number of coronary events and end points have been reduced (Sever et al., 2003).

The CARDS study was designed to assess the effectiveness of cholesterol reduction in the primary prevention of major cardiovascular events in patients with type 2 diabetes without high concentrations of LDL-cholesterol (Colhoun et al., 2004). The study involving 2,838 male and female participants was a randomised, double-blind, placebo-controlled multicentre trial conducted in the UK. All the participants had type 2 diabetes and at least one other risk factor for cardiovascular disease. Their serum LDL-cholesterol level was not high (less than $4.14 \mathrm{mmol} \mathrm{L}^{-1}$ with a median of $3.1 \mathrm{mmol} \mathrm{L}^{-1}$ ). The study was terminated earlier than the sheduled follow-up period of four years because 210 events have been recorded. There was a reduction in major cardiovascular events, acute coronary events and stroke by $37 \%$ (absolute risk reduction 3.2\%; $\mathrm{p}=0.001$ ), 36\% (absolute risk reduction 1.9\%; $\mathrm{p}=$ 0.013 ) and $48 \%$ (absolute risk reduction 1.3\%; $\mathrm{p}=$ 0.016) respectively compared with placebo when patients are treated daily with atorvastatin $10 \mathrm{mg}$. It is noteworthy that the this benefits were observed independent of the entry LDL-cholesterol or triglyceride levels at the start of the study (Colhoun et al., 2004).

\subsection{Mixed Secondary and Primary Prevention Trials}

The Heart Protection Study (HPS) enrolled a total of 20,536 patients out of which 5,963 were diabetic patients (HPSCG, 2002). The participants were randomly allocated to simvastatin or placebo. Coronary deaths was reduced by $20 \%$, major coronary events had a reduction of $27 \%$ and first revascularisation was also reduced by $17 \%$ in the diabetic group receiving simvastatin. This further strengthen the fact that cholesterol-lowering therapy is beneficial in people with diabetes mellitus whether or not they have elevated cholesterol level (HPSCG, 2002). The HPS also revealed the effect of cholesterol-lowering therapy on other diabetes-related outcomes. Macrovascular complications were significantly reduced inthe diabetic group. There were fewer leg ulcers and amputations (Collins et al., 2003).

The Atorvastatin Study for Prevention of Coronary Heart Disease End Points (ASPEN) trial assesses the effect of $10 \mathrm{mg}$ of atorvastatin versus placebo on Cardiovascular Disease (CVD) prevention in subjects with type 2 diabetes and LDL cholesterol levels below contemporary guideline targets (Knopp et al., 2006). The ASPEN trial was a randomised, double-blind, parallelgroup study. The composite primary end point comprised cardiovascular death, nonfatal myocardial infarction, nonfatal stroke, recanalization, coronary artery bypass surgery, resuscitated cardiac arrest and worsening or unstable angina requiring hospitalization. Randomization was done with 2,410 patients with type 2 diabetes mellitus. The outcome of the study showed that there was no statistically significant reduction in the composite end point. It is however noteworthy to note that the procol for the ASPEN study changed because of changing treatment guidelines. For this reason, the ASPEN study was unable to confirm the benefit of statin therapy but was nevertheless be able to show a mean LDL cholesterol reduction in the atorvastatin group over 4 years was $29 \%$ versus placebo $(\mathrm{p}<0.0001)$ (Knopp et al., 2006).

The Diabetes and Dialysis Study is a multicenter, randomized, double-blind, prospective study of 1255 subjects with type 2 diabetes mellitus. These patients were on maintenance hemodialysis. They were randomly assigned to receive $20 \mathrm{mg}$ of atorvastatin per day or matching placebo. The primary end point was a composite of death from cardiac causes, nonfatal myocardial infarction and stroke. Secondary end points included death from all causes and all cardiac and cerebrovascular events combined (Wanner et al., 2005). Atorvastatin had no significant effect on the composite primary end point of cardiovascular death, nonfatal MI and stroke (Wanner et al., 2005). The inability of this study to show the benefit of statin therapy in diabetic patients undergoing maintenance haemodialysis may be due to the fact that patients on haemodialysis have other factors contributing to cardiovascular risk (Baigent et al., 2000). Patients with end-stage renal disease have an inverse associations between blood cholesterol (as well as other risk factors including hypertension and obesity) and all-cause or cardiovascular mortality (Baigent et al., 2000; Brady and Betteridge, 2003). 


\subsection{Other Studies}

There are other studies involving diabetic patients and the use of statins in the prevention of cardiovascular risk. These studies however seek to evaluate whether intensive or moderate lipid lowering with statins will prevent cardiovascular risk and to what extent.

The PROVE-IT study sets out to determine if reducing LDL-Cholesterol below the level recommended by the guidelines confers any further benefit on the patient (Cannon et al., 2004). The PROVE-IT study enrolled 4162 patients who had been hospitalized for an acute coronary syndrome and compared $40 \mathrm{mg}$ of pravastatin daily (standard therapy) with $80 \mathrm{mg}$ of atorvastatin daily (intensive therapy). Diabetic patient constitutes $18 \%$ of the enrolled subjects. There was a significant reduction in LDL-cholesterol level in the high-dose atorvastatin group $\left(95 \mathrm{mg} \mathrm{dL}^{-1}\right.$ in the pravastatin group and $62 \mathrm{mg} \mathrm{dL}{ }^{-1}$ in the atorvastatin group). The benefit of high dose atorvastatin on the primary end point death from any cause, myocardial infarction, documented unstable angina requiring rehospitalization, revascularization and stroke was established (Cannon et al., 2004).

The Treating-to-New-Targets (TNT) study was a prospective, double blind, parallel-group trial. 10,001 patients with clinically evident coronary heart disease patients were enrolled (Deedwania et al., 2006). Of the 10,001 patients, 5584 patients had metabolic syndrome based on the 2005 NCEP ATP III criteria and 1,231 patient had diabetes. Patients were randomly assigned to receive either atorvastatin $10 \mathrm{mg}$ per day $(\mathrm{n}=2820)$ or $80 \mathrm{mg}$ per day $(\mathrm{n}=2764)$. The primary outcome measure was time to first major cardiovascular event, defined as death from coronary heart disease, non-fatal nonprocedure-related myocardial infarction, resuscitated cardiac arrest, or fatal or non-fatal stroke. LDLcholesterol was lowered to $99 \mathrm{mg} \mathrm{dL}^{-1}$ in those that received $10 \mathrm{mg}$ atorvastatin and $72 \mathrm{mg} \mathrm{dL}^{-1}$ in those that received $80 \mathrm{mg}$ of atorvastatin (Deedwania et al., 2006). High-dose (compared with low-dose) atorvastatin reduced the risk of the primary end point (time to first major cardiovascular event) in diabetic patients, with a relative risk reduction similar to that observed in nondiabetic patients (Deedwania et al., 2006).

The A-Z Trial compared early intensive versus a delayed conservative simvastatin treatment in patients with acute coronary syndromes. Patients with diabetes were $24 \%$ of the study population. The LDL cholesterol level achieved was $63 \mathrm{mg} \mathrm{dL} \mathrm{d}^{-1}$ in the early intensive treatment and $77 \mathrm{mg} \mathrm{dL}^{-1}$ in the delayed conservative treatment (Lemos et al., 2004). In this study, the intensive strategy did not significantly reduce the risk for the composite end point of cardiovascular death, nonfatal MI, readmission for acute coronary syndrome and stroke and the diabetic subgroup was no exception (Lemos et al., 2004).

\subsection{Risk Assessment}

The benefit of cholesterol lowering with statins is well established for primary and secondary prevention of CHD in non-diabetic patients with both elevated and relatively 'normal' serum cholesterol levels (SSSS, 1994; Sacks et al., 1996; LIPID, 1998; Shepherd et al., 1995; Downs et al., 1998). Three key studies (4S, CARE, LIPID) (SSSS, 1994; Sacks et al., 1996; LIPID, 1998) described above have been able to establish the role of statins in secondary prevention of CHD in diabetic patients. Apart from lowering cholesterol level, statins also have anti-inflammatory actions. The role of inflammation in the development of atherosclerosis have also been established. Using statin for this purpose is an important consideration as a therapy in the nearest future (Brady and Betteridge, 2003).

Two studies with good evidence to support the role of statins in primary prevention for diabetic population are HPS and CARDS (Colhoun et al., 2004; Collins et al., 2003). The two studies have almost the same number of primary prevention patients and both were were able to establish a statistical difference within this group. They included diabetic patients with few other CHD risk factors and still showed benefit within this subgroup (Colhoun et al., 2004; Collins et al., 2003).

It has been well established that all diabetic patients are not the same (EPDETHBCA, 2001). The NCEP ATP III guidelines recognized this fact (EPDETHBCA, 2001). The assessment of the level of CHD risk in people with diabetes as presently obtains, using the risk estimation table, is not adequately done. Some patient may thus be left out of statin and antihypertensive treatments for primary prevention They also persistently underestimate the level of risk. There are many reasons for this, including the fact these tables are based on Framingham data which had few diabetic subjects (Winocour and Fisher, 2003).

The high incidence of CHD in people with diabetes has led many to believe that diabetes should be considered as a CHD risk equivalent and therefore all 
patients should be given a statin (Turner et al., 1998). In Finland, patients with previous MI who are diabetic have similar CHD events when compared with patients with MI who are not diabetic (Haffner et al., 1998). In a study from Tayside, people with diabetes without CHD had a higher event rate than in non-diabetic people without CHD. The event rate in non-diabetic subjects with previous MI was however higher than in those with diabetes with previous MI (Evans et al., 2002). In HPS, which is primary prevention study, risk of CHD events in diabetic patients was lower than the risk in the secondary prevention subjects in $4 \mathrm{~S}, \mathrm{CARE}$ and LIPID. From the foregoing, it is an established fact that there is a higher risk of CHD in patients with diabetes mellitus. The question does arise-should all people with diabetes be treated with statin therapy?

\subsection{Should all Patients with Diabetes Mellitus be on Statin Treatment?}

A log-linear relation exists between blood LDL cholesterol and the risk of CHD and this association persists well below the range of typical cholesterol levels (Jacobs et al., 1992; Chen et al., 1991; Kearney et al., 2008). There still remains a substantial relative risk even when LDL is adequately lowered and so LDL reduction though beneficial is not a panacea to cardiovascular risk in DM.

\section{CONCLUSION}

We are of the opinion that treatment decisions should be based on the reduction of absolute risk and not on the reduction in relative risk. Treatment decisions should be based on the reduction of absolute risk and not on the reduction in relative risk. Patients suffering from diabetes mellitus will incur a huge cost if they were to be commenced on statin from the day the diagnosis of diabetes was made. With the rising incidence of diabetes worldwide, statins should be targeted to those patients with diabetes mellitus who are most likely to benefit from it. It might suffice to suggest that type $2 \mathrm{DM}$ patients with metabolic syndrome or those with mild to moderate cardiovascular risk will benefit from statin therapy. Clinical benefits stand to be derived from statin therapy if moderate reduction of risk can be achieved in patients that are classified as having high cardiovascular risk. What this means is that all patients eligible to be enrolled in the HPS or CARDS study should be on statin treatment.

\section{REFERENCES}

Baigent, C., K. Burbury and D. Wheeler, 2000. Premature cardiovascular disease in chronic renal failure. Lancet, 356: 147-152. DOI: 10.1016/S01406736(00)02456-9

Brady, A.J.B. and D.J. Betteridge, 2003. Prevalence and risks of undertreatment with statins. Br. J. Cardiol., 10: 218-19.

Cannon, C.P., E. Braunwald, C.H. McCabe, D.J. Rader and J.L. Rouleau et al., 2004. Intensive versus moderate lipid lowering with statins after acute coronary syndromes. N. Engl. J. Med., 350:14951504. DOI: 10.1056/NEJMoa040583

Chen, Z., R. Peto, R. Collins, S. MacMahon and J. Lu et al., 1991. Serum cholesterol concentration and coronary heart disease in population with low cholesterol concentrations. BMJ, 303: 276-282.

Colhoun, H.M., D.J. Betteridge, P.N. Durrington, G.A. Hitman and H.A. Neil et al., 2004. Primary prevention of cardiovascular disease with atorvastatin in type 2 diabetes in the Collaborative Atorvastatin Diabetes Study (CARDS): Multicentre randomised placebo-controlled trial. Lancet, 364: 685-96. PMID: 15325833

Collins, R., J. Armitage, S. Parish, P. Sleigh and R. Peto et al., 2003. MRC/BHF Heart Protection Study of cholesterol-lowering with simvastatin in 5963 people with diabetes: A randomised placebocontrolled trial. Lancet, 361: 2005-2016. PMID: 12814710

Graaf, J.D., H.L. Hak-Lemmers, M.P. Hectors, P.N. Demacker and J.C. Hendriks et al., 1991. Enhanced susceptibility to in vitro oxidation of the dense low density lipoprotein subfraction in healthy subjects. Arterioscler. Thromb., 11: 298-306. PMID: 1998647

Lemos, J.A.D., M.A. Blazing, S.D. Wiviott, E.F. Lewis and K.A.A. Fox et al., 2004. Early intensive Vs a delayed conservative simvastatin strategy in patients with acute coronary syndromes: Phase $\mathrm{Z}$ of the A to $\mathrm{Z}$ trial. JAMA, 292: 1307-1316. DOI: 10.1001/jama.292.11.1307

Deedwania, P., P. Barter, R. Carmena, J.C. Fruchart and S.M. Grundy et al., 2006. Reduction of low-density lipoprotein cholesterol in patients with coronary heart disease and metabolic syndrome: Analysis of the Treating to New Targets study. Lancet, 368: 919-928. DOI: 10.1016/S0140-6736(06)69292-1 
Downs, J.R., M. Clearfield, S. Weiss, E. Whitney and D.R. Shapiro et al., 1998. Primary prevention of acute coronary events with lovastatin in men and women with average cholesterol levels: Results of AFCAPS/TexCAPS. Air force/texas coronary atherosclerosis prevention study. JAMA, 279: 161522. PMID: 9613910

Evans, J.M.M., J. Wang and A.D. Morris, 2002. Comparison of cardiovascular risk between patients with type 2 diabetes and those who had had a myocardial infarction: Cross sectional and cohort studies. BMJ, 324: 939-942. PMID: 11964337

EPDETHBCA, 2001. Executive Summary of the Third Report of the National Cholesterol Education Program (NCEP). JAMA, 285: 2486-2497. DOI: 10.1001/jama.285.19.2486

Ford, E.S., W.H. Giles and W.H. Dietz, 2002. Prevalence of the metabolic syndrome among US adults: Findings from the Third National Health and Nutrition Examination Survey. JAMA, 287: 356359. PMID: 11790215

Goldberg, R.B., M.J. Mellies and F.M. Sacks, L.A. Moyé and B.V. Howard et al., 1998. Cardiovascular events and their reduction with pravastatin in diabetic and glucose-intolerant myocardial infarction survivors with average cholesterol levels: Subgroup analyses in the Cholesterol And Recurrent Events (CARE) Trial. The care investigators. Circulation, 98: 2513-19. PMID: 9843456

Haffner, S.M., S. Lehto, T. Rönnemaa, K. Pyörälä and M. Laakso, 1998. Mortality from coronary heart disease in subjects with type 2 diabetes and in nondiabetic subjects with and without prior myocardial infarction. N. Engl. J. Med., 339:229-34. PMID: 9673301

Haffner, S.M., M.P. Stern, H.P. Hazuda, B.D. Mitchell and J.K. Patterson, 1990. Cardiovascular risk factors in confirmed prediabetic individuals. Does the clock for coronary heart disease start ticking before the onset of clinical diabetes? JAMA, 263: 2893-2898. PMID: 2338751

Haffner, S.M., 2004. Dyslipidemia management in adults with diabetes. Diabetes Care, 27: S68-S71. PMID: 14693930

Haffner, S.M., 1998. Management of dyslipidemia in adults with diabetes. Diabetes Care, 21: 160-178. PMID: 9538988
HPSCG, 2002. MRC/BHF Heart Protection Study of cholesterol lowering with simvastatin in 20,536 high-risk individuals: A randomised placebocontrolled trial. Lancet, 360: 7-22. PMID: 12114036

IDF, 2012. Diabetes atlas. International Diabetes Federation.

Jacobs, D., H. Blackburn, M. Higgins, D. Reed and H. Iso et al., 1992. Report of the conference on low blood cholesterol: Mortality associations. Circulation, 86: 1046-1060. PMID: 1355411

Kearney, P.M., L. Blackwell, R. Collins, A. Keech and J. Simes et al., 2008. Efficacy of cholesterol-lowering therapy in 18,686 people with diabetes in 14 randomised trials of statins: A meta-analysis. Lancet, 371: 117-125. DOI: 10.1016/S01406736(08)60104-X, PMID: 18191683

Keech, A., D. Colquhoun, J. Best, A. Kirby and R.J. Simes et al., 2003. Secondary prevention of cardiovascular events with long-term pravastatin in patients with diabetes or impaired fasting glucose: Results from the LIPID trial. Diabetes Care, 26: 2713-2721. PMID: 14514569

Knopp, R.H., M. d'Emden, J.G. Smilde, S.J. Pocock, 2006. Efficacy and safety of atorvastatin in the prevention of cardiovascular end points in subjects with type 2 diabetes: The Atorvastatin Study for Prevention of coronary heart disease Endpoints in Non-insulin-dependent diabetes Mellitus (ASPEN). Diabetes Care, 29:1478-1485. DOI: 10.2337/dc052415

Mokdad, A.H., E.S. Ford, B.A. Bowman, W.H. Dietz and F. Vinicor et al., 2003. Prevalence of obesity, diabetes and obesity-related health risk factors, 2001. JAMA, 289: 76-79. PMID: 12503980

Ogbera, A.O., O.A. Fasanmade, S. Chinenye and A. Akinlade, 2009. Characterization of lipid parameters in diabetes mellitus--a Nigerian report. Int. Arch. Med., 2: 19-19. PMID: 19619328

Pyorala, K., T.R. Pedersen, J. Kjekshus, O. Faergeman and A.G. Olsson et al., 1997. Cholesterol lowering with simvastatin improves prognosis of diabetic patients with coronary heart disease. A subgroup analysis of the Scandinavian Simvastatin Survival Study (4S). Diabetes Care, 20: 614-20. PMID: 9096989

Sacks, F.M., M.A. Pfeffer, L.A. Moye, J.L. Rouleau and J.D. Rutherford et al., 1996. The effect of pravastatin on coronary events after myocardial infarction in patients with average cholesterol levels. Cholesterol and recurrent events trial investigators. N. Engl. J. Med., 335: 1001-1009. PMID: 8801446 
Sever, P.S., B. Dahlof, N.R. Poulter, H. Wedel and G. Beevers et al., 2003. Prevention of coronary and stroke events with atorvastatin in hypertensive patients who have average or lower-than-average cholesterol concentrations, in the Anglo-Scandinavian Cardiac Outcomes Trial--Lipid Lowering Arm (ASCOT-LLA): A multicentre randomised controlled trial. Lancet, 361: 1149-58. PMID: 12686036

Shepherd, J., S.M. Cobbe, I. Ford, C.G. Isles and A.R. Lorimer et al., 1995. Prevention of coronary heart disease with pravastatin in men with hypercholesterolemia. N. Engl. J. Med., 333: 130107. PMID: 7566020

LIPID, 1998. Prevention of cardiovascular events and death with pravastatin in patients with coronary heart disease and a broad range of initial cholesterol levels. N. Engl. J. Med., 339: 1349-57. PMID: 9841303
SSSS, 1994. Randomised trial of cholesterol lowering in 4444 patients with coronary heart disease: The Scandinavian Simvastatin Survival Study (4S). Lancet, 344: 1383-1389. PMID: 7968073

Turner, R.C., H. Millns, H.A, Neil, I.M. Stratton and S.E. Manley et al., 1998. Risk factors for coronary artery disease in non-insulin dependent diabetes mellitus: United kingdom prospective diabetes study (UKPDS: 23). BMJ, 316: 823-828.

Wanner, C., V. Krane, W. Marz, M. Olschewski, J.F. Mann, G. Ruf et al., 2005. Atorvastatin in patients with type 2 diabetes mellitus undergoing hemodialysis. N. Engl. J. Med., 353: 238-248. DOI: 10.1056/NEJMoa043545

Winocour, P.H. and M. Fisher, 2003. Prediction of cardiovascular risk in people with diabetes. Diabet. Med., 20: 515-527. PMID: 12823231 